\title{
Temperature and flow fields in a high prandtl number liquid bridge under microgravity
}

\author{
Shuo Yang ${ }^{1, a}$, Ruquan Liang ${ }^{1}$, Jicheng $\mathrm{He}^{1}$ \\ ${ }^{1}$ Key Laboratory of National Education Ministry for Electromagnetic Processes of Materials, Northeastern University in Shen Yang, China
}

\begin{abstract}
The temperature and flow fields of high prandtl number liquid bridge with surface deformation have been investigated under microgravity by a developed numerical model, and numerical simulations have been carried out based on the Navier-Stokes equations coupled with the energy conservation equation on a staggered grid. In numerical calculations, the free surface deformation and the effects of ambient air are considered. The surface deformation of liquid bridge is monitored by level set method of mass conservation to capture two phase interfaces. Simultaneously, results of temperature and flow fields in liquid bridge are given.
\end{abstract}

\section{Introduction}

Convective motion induced by local variations of surface tension along a liquid free surface is called Marangoni convection. Although such variations can be caused by differences in temperature or composition, our main interest here is temperature-induced convection, which is often called thermocapillary convection. The floating zone convection is one kind of thermocapillary convection. There have been many researches and applications of crystal growing by floating zone method on the ground, while the size of the crystal growing in floating zone is limited on the ground. However, the size of the crystal growing is improved under space environment. The study on the characteristics of thermocapillary convection in the floating zone has become an important issue in order to optimize the crystal growing.

Despite a lot of experiments and numerical simulations on the half-floating zone method have been carried out, almost all studies did not consider the effects of ambient air for thermocapillary convection mechanism. Recently, some scholars have conducted studies to investigate flow and temperature fields in liquid bridges involving the effects of ambient air. Tiwari and Nishino ${ }^{[1]}$ carried out the research regarding the effects of ambient air temperature on the surface heat transfer characteristics of a liquid bridge by using the "panel" boundary condition. Lrikura et al. ${ }^{[2]}$ studied the effects of ambient air on critical parameters of a liquid bridge using numerical and experimental methods. Although the effect of the ambient air was considered, the surface deformation of the liquid bridge was not considered in their studies. In addition, the flow characteristic has been still in debate for liquid bridges of high $\operatorname{Pr}$ number $(\operatorname{Pr}>15)$ fluid. So many scholars have conducted related experiments and numerical simulations. Levenstam and Amberg ${ }^{[3]}$ investigated the lost steady process of the surface thermocapillary convection in a cylindrical liquid bridge for low $\operatorname{Pr}$ number $(\mathrm{Pr}=0.01)$ fluid with aspect ratio $H / R=1$. They proposed that the two-dimension steady axisymmetric convection firstly changed for three dimensional steady convection with Reynolds number increasing $(\mathrm{Re}=1960)$. When $\mathrm{Re}$ number was 6250, the three dimensional steady convection further changed into the oscillation convection. Bazzi et al. ${ }^{[4]}$ investigated the stability of the thermocapillary convection in a liquid bridge of low $\operatorname{Pr}$ number $(\operatorname{Pr}=0.016)$ fluid with aspect ratio $H / R=0.7$. Their research demonstrated that when Marangoni number was 48 , the first time instability happened, and then the second time instability happened corresponding to $\mathrm{Ma}=80$. For high $\operatorname{Pr}$ number $(\operatorname{Pr}>15)$ fluid, there was still contention on the flow characteristics of liquid bridges.

A mass conserving level set method for monitoring the two-phase interface has been used in this paper. The surface deformation of liquid bridge and effects of ambient air are all taken into consideration in the numerical calculations. The complete numerical simulations for liquid bridge of high $\operatorname{Pr}$ number fluid under microgravity are offered.

\section{Physical model}

The simplified model of floating-zone crystal growing is adopted in the present paper as shown in figure 1. The diameter of upper and bottom discs is $D=5.00 \mathrm{~mm}$. The

${ }^{a}$ S. Yang: 812886674@qq.com 
aspect ratio $(H / R)$ is 1 . Other main physical parameters are presented in Fig. 1.

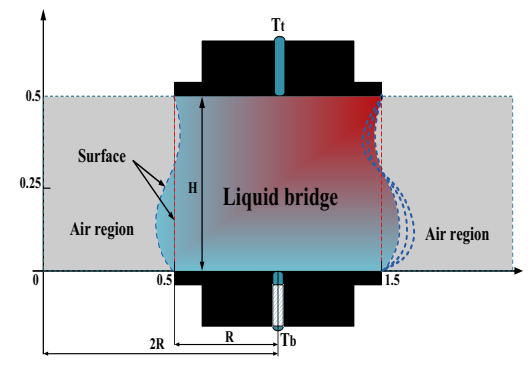

$R$ : Radius of liquid bridge.

$H$ : Height of liquid bridge.

$T_{t}, T_{b}$ : Temperature of upper and bottom

disks, respectively.

$$
\Delta T=T_{t}-T_{b}
$$

$2 R$ : Outer diameter of gas-phase region.

Fig 1. Schematic of a half-zone liquid bridge model

\section{Controlling equations}

The surface tension on the free interface is a linear function of temperature and decreases linearly with the temperature increasing. The basic governing equations of the half-floating zone thermocapillary convection include mass conservation, momentum conservation, and energy conservation equations. The non-dimensional governing equations are given as following:

$$
\begin{gathered}
\mathbf{u}_{t}+(\mathbf{u} \cdot \nabla) \mathbf{u}= \\
\frac{1}{\rho}\left(-\nabla p+\frac{1}{\operatorname{Re}} \nabla \cdot(2 \mu \mathbf{D})+\left(\frac{1}{\mathrm{We}}-\frac{\mathrm{Ca}}{\mathrm{We}} \Theta\right) \kappa \delta(d) \mathbf{n}\right) \\
\nabla \cdot \mathbf{u}=0 \\
\frac{\partial \Theta}{\partial t}+\nabla \cdot(\mathbf{u} \Theta)=\frac{1}{\operatorname{Re} \operatorname{Pr}} \nabla^{2} \Theta
\end{gathered}
$$

where $\mathbf{u}=(u, v)$ is the fluid velocity, $\rho=\rho(\mathbf{x}, t)$ is the fluid density, $\mu=\mu(\mathbf{x}, t)$ is the fluid viscosity, $\mathrm{D}$ is the viscous stress tensor, $\kappa$ is the curvature of the interface, $d$ is the normal distance to the interface, $\delta$ is the Dirac delta function, $\mathrm{n}$ is the unit normal vector at the interface, $\mathrm{t}$ is the time, $\mathrm{p}$ is the pressure.

The key parameters are $\operatorname{Re}=\rho_{l} U L / \mu_{l}$, Reynolds number, where $\mathrm{L}$ is the characteristic length defined as $L=2 R$, where $\mathrm{R}$ is the initial radius of the liquid bridge. $U$ is the characteristic velocity defined as $U=\sigma_{T} \Delta T / \mu_{l}$ in microgravity conditions, where $\sigma_{T}$ is the temperature dependency of surface tension, $\Delta T=T_{t}-T_{b}$ is the temperature difference between the top and bottom disks ; We $=U^{2} L \rho_{l} / \sigma$, Weber number; $\operatorname{Pr}=\mu_{l} / \rho_{l} a$, Prandtl number, where $a$ is the thermal diffusivity; $\mathrm{Ca}=\mu_{l} U / \sigma$, Capillary number; We denote $\sigma$ as the surface tension, $\sigma=\sigma_{c}-\sigma_{T}\left(T-T_{b}\right)$, where $\sigma_{c}$ is a reference value of surface tension, and $\Theta$ as the excess temperature, $\Theta=\left(T-T_{b}\right) / \Delta T$. Here $\rho_{l}$ and $\mu_{l}$ are the dimensional liquid density and viscosity, respectively, and the dimensionless density $\left(\rho_{l} / \rho_{l}\right)$ and viscosity $\left(\mu_{l} / \mu_{l}\right)$ inside the liquid bridge are equal to $1 . \rho_{g}$ and $\mu_{g}$ are the dimensional density and viscosity of ambient air, respectively.

\section{Results and discussion}

In the following, the analysis of temperature field in liquid bridge of high Pr number fluid under microgravity is given. The computational conditions are given as follows: the Marangoni number is $8815, \mathrm{M} a=\operatorname{Re} \operatorname{Pr}$, the Prandtl number is 69 , the Weber number is 9.506 , the Capillary number is 0.0745 , and gravity level is $g=0.1 \mathrm{~g}_{0}$ $\left(g_{0}=9.81 \mathrm{~m} / \mathrm{s}^{2}\right)$. Other main calculation parameters are shown in table 1 .

Table 1: Physical parameters

\begin{tabular}{|c|c|}
\hline Element & Size \\
\hline $\begin{array}{c}\text { Temperature of upper disk, } \\
(\mathrm{K})\end{array}$ & 325 \\
\hline $\begin{array}{c}\text { Temperature of bottom disk, } \\
(\mathrm{K})\end{array}$ & 300 \\
\hline Density ratio, $\rho_{l} / \rho_{g}$ & 757.45 \\
\hline Density $\rho_{l},\left(\mathrm{~kg} / \mathrm{m}^{3}\right)$ & 760 \\
\hline Viscosity ratio, $\mu_{l} / \mu_{g}$ & 254.44 \\
\hline Viscosity $\mu_{l},(\mathrm{~Pa} \cdot \mathrm{s})$ & $4.58 \times 10^{-3}$ \\
\hline
\end{tabular}

The time evolution of the temperature field within the liquid bridge is shown in Fig. 2, the thermocapillary convection starts near the hot corner at the initial time, and the vortex center of thermocapillary convection is at the hot corner (see Fig.3). Thus, the initial temperature field has a large temperature gradient at this region. In addition, the horizontal convection is caused by the return flow which uplifts the temperature near the cold disc, and the temperature gradient increases near the cold corner. The vortex center of the thermocapillary convection moves toward the cold disk and interior of the liquid bridge. Eventually, the temperature gradient is reduced at the hot corner, and the temperature distribution tends to be uniform in the inner of liquid bridge. Comparing with the convection heat transfer near the free surface, the 
conductive heat transfer can be found in the inner of liquid bridge. Mreover, the temperature gradient at the cold corner near the free surface is large.

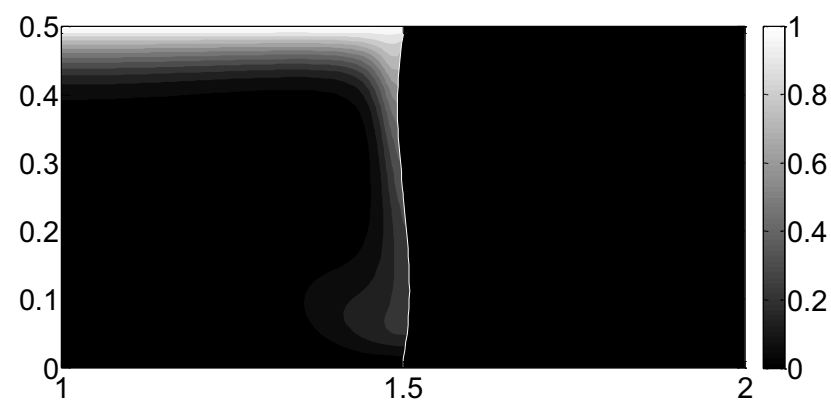

(a) $t=10.5$

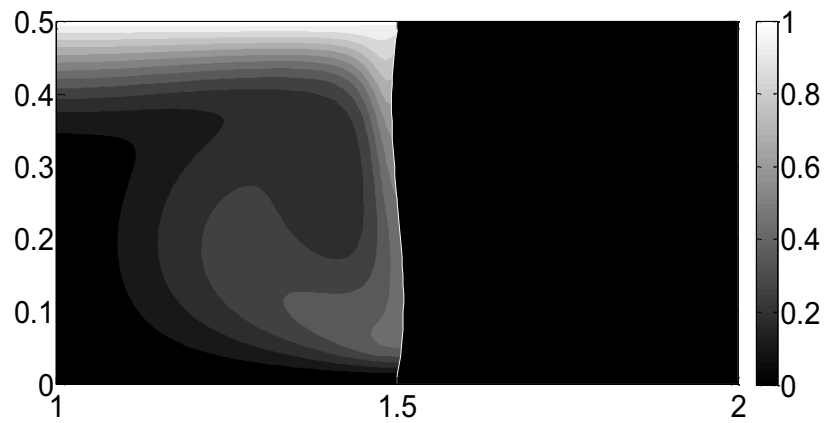

(b) $t=75.0$

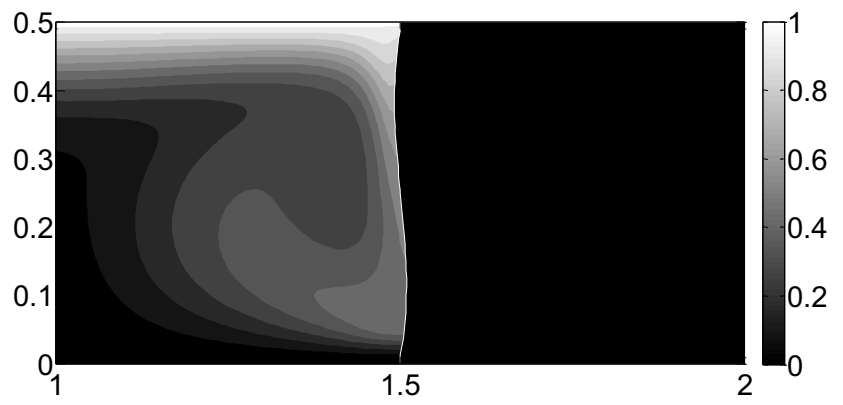

(c) $t=100.0$

Fig 2. Temperature evolution inside the liquid bridge under $0.1 \mathrm{~g}_{0}$ gravity condition

( $P r=69, M a=8815, W e=8815, C a=0.0745, D=5.0 \mathrm{~mm}, H / R=1.0$, $\left.g=0.1 g_{0}\right)$

\section{Conclusions}

1. In the initial period, the thermocapillary convection originates from the hot corner. The temperature gradient is relatively large at there.

2. As the development of the thermocapillary convection, the vortex center of thermocapillary convection moves from the hot corner to the cold corner, therefore, the temperature gradient at hot corner is reduced generally and the temperature gradient begins to increase at cold corner.

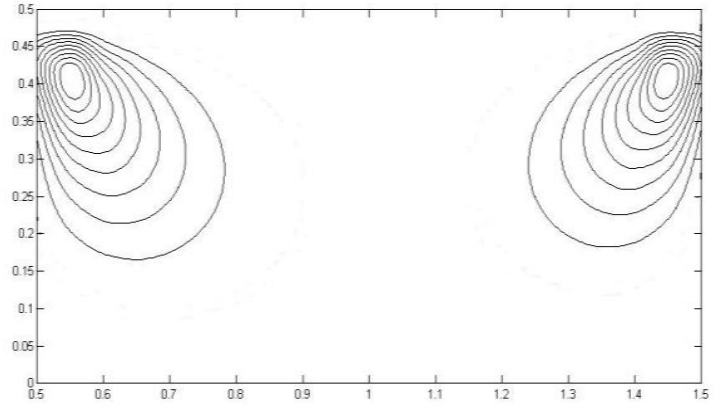

(a) $t=2.5$

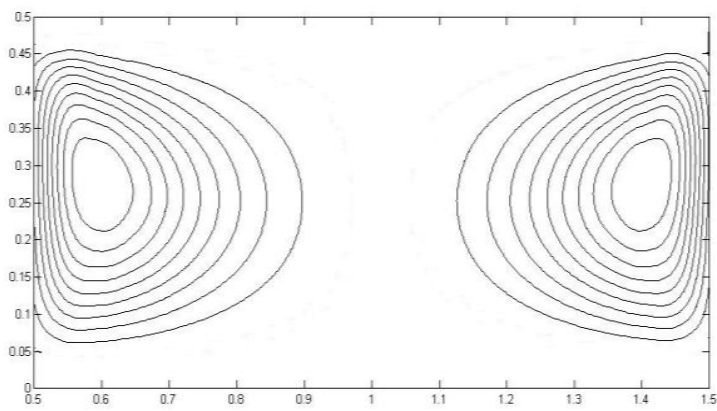

(b) $t=100$

Fig 3. Streamlines in the liquid bridge at different time $(P r=69, M a=8815, W e=8815, C a=0.0745, D=5.0 \mathrm{~mm}, H / R=1.0$, $\left.g=0.1 g_{0}\right)$

3. The temperature distribution tends to be uniform in the inner of liquid bridge due to the action of horizontal convection, but the temperature gradient is still lagre near the free surface.

\section{Acknowledgements}

The present work is supported financially by the National Natural Science Foundation of China under the grants of 51376040 and 11072057.

\section{References}

1. Tiwari S., Nishino K, J. Cryst. Growth., Numerical study to investigate the effect of partition block and ambient air temperature on interfacial heat transfer in liquid bridges of high prandtl number fluid. 2, 486-496, (2007)

2. Irikura M., Arakawa Y., Ueno I. \& Kawamura H. Microgravity. Sci. Tec, Effect of ambient fluid flow upon onset of oscillatory thermocapillary convection in half-zone liquid bridge. 16, 176-180 (2005)

3. Levenstam M, Amberg G, J. Fluid. Mech., Hydrodynamical instabilities of thermocapillary flow in a half-zone. 297, 357-372 (1995)

4. Bazzi H., Nguyen C. \& Galanis N, Int. J. Therm.Sci, Numerical study of the unstable thermocapillary flow in a silicon float zone under $\mu-g$ condition. 8,702-716 (2001) 